\title{
TIPS: Lieber früher als später
}

Bei Patienten mit akuter Ösophagusvarizenblutung geht das frühzeitige Einsetzen eines transjugulären intrahepatischen portosystemischen Shunt (TIPS) mit einer Reduktion des Therapieversagens und der Mortalität einher.

- Patienten mit Leberzirrhose Child B und $\mathrm{C}$ und trotz endoskopischer Maßnahmen anhaltender Ösophagusvarizenblutung haben eine besonders schlechte Prognose. Der massive Blutanfall im Intestinum führt in der Regel zu einer deutlichen Verschlechterung der Leberfunktion und der hepatischen Enzephalopathie. Daher ist man mit dem Einsatz eines TIPS in der Regel zurückhaltend, da diese Maßnahme ihrerseits zu einem weiteren Ammoniakanstieg und einer Zunahme der hepatischen Enzephalopathie beiträgt.

In einer randomisierten Studie an 63 Patienten mit fortgeschrittener Leberzirrhose und akuter Varizenblutung, die mit vasoaktiven Medikamenten und endoskopischer Therapie behandelt wurden, implantierte man innerhalb von 72 Stunden einen TIPS oder führte nur die konservative und endoskopische Behandlung weiter fort. Innerhalb eines medianen Follow-up von 16 Monaten kam es nur bei einem Patienten in der TIPS-Gruppe zu einer erneuten Blutung bzw. zum Versagen der Blutstillung.

Die 1-Jahres-Wahrscheinlichkeit des Nichtauftretens einer erneuten Blutung lag in der pharmakologisch behandelten Gruppe bei 50\%, in der Gruppe mit der frühzeitigen TIPS-Implantation dagegen bei 97\%. 16 Patienten verstarben innerhalb des Beobachtungszeitraums, davon zwölf in der Kontroll-, dagegen nur vier in der Studiengruppe.

\section{Kommentar}

Die Untersuchung zeigt ein sehr positives Ergebnis der frühen TIPS-Anlage bei diesen schwer kranken Patienten. Allerdings wurde das Ergebnis mit einem
4 Ösophagusvarizenblutung.

() Arteria Photography

technischen Aufwand erzielt, der gerade in der Notfallsituation nicht in allen Krankenhäusern zur Verfügung steht. Die Weite des Shunts, die initial $8 \mathrm{~mm}$ betrug, wurde nämlich anhand des portokavalen Druckgradienten gewählt. Wenn dieser mit dem 8- $\mathrm{mm}$ Shunt nicht unter $12 \mathrm{mmHg}$ sank, dilatierte man den Shunt auf $10 \mathrm{~mm}$. Es ist auch erstaunlich, dass es in der frühzeitig mit TIPS behandelten Gruppe nicht zu einer Zunahme des Schweregrads einer hepatischen Enzephalopathie kam. Die Untersuchung leistet sicher einen wertvollen Beitrag für die Akutbehandlung von Patienten mit Ösophagusvarizenblutung. Ob sich die aufwendige Technik in allen Kliniken durchsetzt, ist sehr fraglich.

H. S. FüEßL =

- J. C. Garcia-Pagán et al.

Early use of TIPS in patients with cirrosis and variceal bleeding. New England J. Med. 362 (2010) 25, 2370-2379.

\section{Melatonin gegen Schlafstörungen bei älteren Menschen}

\author{
Ein Schlafmittel mit wenig Nebenwirkungen, das auch \\ bedenkenlos über einen längeren Zeitraum gegeben \\ werden kann - das gibt es anscheinend. Es wirkt aber nur \\ bei Senioren.
}

- Ein Team britischer und israelischer Forscher rekrutierte 791 Patienten mit primärer Insomnie. Diese erhielten $30 \mathrm{Wo-}$ chen lang entweder Placebo oder $2 \mathrm{mg}$ Melatonin (slow release), das 1-2 Stunden vor dem Schlafengehen eingenommen werden sollte.

Die Auswertungen zeigen, dass insbesondere Personen über 65 Jahre gut auf die Therapie ansprechen. Der Effekt ist sowohl kurz- wie auch langfristig signifikant. Nebenwirkungen waren kaum zu beklagen. Nach dem Absetzen der Therapie wurde kein Reboundphänomen beobachtet. Die Autoren meinen daher, dass die Melatonintherapie älterer, schlafgestörter Patienten wirksam und sicher sei.

\section{Kommentar}

Dies ist eine gut gemachte Studie, die viele interessante Ergebnisse liefert. Sie klärt einige der Widersprüche, die in Vorstudien zu Melatonin aufgetaucht waren. Insbesondere scheint die Erkenntnis wichtig, dass nur ältere Menschen auf diese Behandlung ansprechen, und dass selbst bei längerer Therapie kaum mit Nebenwirkungen zu rechnen ist. Bedenkt man die nicht unerheblichen Risiken herkömmlicher Schlafmittel, erscheint Melatonin bei schlafgestörten Senioren einen Versuch wert zu sein.

E. ERNST =

\section{- A. G. Wade et al.}

Nightly treatment of primary insomnia with prolonged release melatonin for 6 months: a randomized placebo controlled trial on age and endogenous melatonin as predictors of efficacy and safety. BMC Med. 16 (2010) 51. 